\title{
THEOREMS ON ASSOCIATION OF VARIABLES IN MULTIDIMENSIONAL LAPLACE TRANSFORMS
}

\author{
JOYATI DEBNATH and NARAYAN CHANDRA DEBNATH \\ Department of Mathematics \\ and Computer Science \\ University of Wisconsin, River Falls \\ River Falls, Wisconsin 54022, U.S.A. \\ (Received May 1, 1988 and in revised form Dcember 2, 1988)
}

ABSTRACT. The inverse of the multidimensional Laplace transform is often obtained by the method of association of variables. In this paper, some basic theorems are developed for evaluating the associated transform of certain types of transformed functions. Many useful associated pairs can be produced with the aid of these fundamental theorems. Several illustrative examples are included.

KEY WORDS AND PHRASES. Multidimensional Laplace transforms, association of variables, associated pair and associated transform.

1980 AMS SUBJECT CLASSIFICATION CODE: $44 A 30$

\section{INTRODUCTION.}

In non-linear systems analysis, multidimensional Laplace transform is applied to solve Volterra model. The special technique often used for the inverse Laplace transform solution is known as the association of variables. Suppose $F\left(s_{1}, s_{2}, \ldots, s_{n}\right)$ be a Laplace transform. Its $n$-dimensional inverse is given by the integral

$$
\begin{aligned}
f\left(t_{1}, t_{2}, \ldots, t_{n}\right) \equiv L_{n}^{-1}\left[F\left(s_{1}, s_{2}, \ldots, s_{n}\right) ; t_{1}, t_{2}, \ldots, t_{n}\right] \\
=\frac{1}{(2 \pi i)^{n}} \int_{\alpha_{1}-i \infty}^{\alpha_{1}+i \infty} \ldots \int_{\alpha_{n}-i \infty}^{\alpha_{n}+i \infty} \exp \left(\sum_{j=1}^{n} s_{j} t_{j}\right) \\
\cdot F\left(s_{1}, s_{2}, \ldots, s_{n}\right) d s_{1} d s_{2} \ldots d s_{n}
\end{aligned}
$$

In certain types of systems analysis, particularly in Volterra series applications [1-2] on non-linear systems [3-5], it becomes essential to invert the $n$-dimensional Laplace transform and specify the inverse image at a single variable, $t$. We denote this image function of one variable as

$$
g(t)=\left.f\left(t_{1}, t_{2}, \ldots, t_{n}\right)\right|_{t_{1}=t_{2}=\ldots=t_{n}=t}
$$

One apprcach to obtain the time function, $g(t)$, is to associate with $F\left(s_{1}, s_{2}, \ldots, s_{n}\right)$ a function $G(s)$ from which an application of the one-dimensional inverse transform 
yields $g(t)$. This particular approach is called the Association of Variables. The function $G(s)$ is said to be the associated transform of $F\left(s_{1}, s_{2}, \ldots, s_{n}\right)$.

Chen and Chiu [6] and Koh [7] have presented several theorems for evaluating $G(s)$ for certain types of $F\left(s_{1}, s_{2}, \ldots, s_{n}\right)$. In this paper, some additional theorems are developed. Few examples are also included for each theorem. However, once the fundamental theorems are established, it is possible to derive as many associated pairs as one desires, and use them flexibly.

\section{THEOREMS ON ASSOCIATION OF VARIABLES}

Suppose $G(s)$ be the associated transform of $F\left(s_{1}, s_{2}, \ldots, s_{n}\right)$ and $G_{1}(s)$ be that of $F\left(s_{1}, s_{2}, \ldots, s_{m-1}, s_{m+1}, \ldots, s_{n}\right), m \leq n$. Let $k$ be any constant, and we restrict the variables $s, s_{1}, s_{2}, \ldots, s_{n}$ to the right half of the complex plane.

Theorem 2.1. If a given function $F\left(s_{1}, s_{2}, \ldots, s_{n}\right)$ can be written in the form

$$
F\left(s_{1}, s_{2}, \ldots, s_{n}\right)=\frac{k}{s_{m}\left(s_{m}+a\right)} F_{1}\left(s_{1}, s_{2}, \ldots, s_{m-1}, s_{m+1}, \ldots, s_{n}\right)
$$

and if $F_{1}\left(s_{1}, s_{2}, \ldots, s_{m-1}, s_{m+1}, \ldots, s_{n}\right) \stackrel{A_{n-1}}{\longrightarrow} G_{1}(s)$. Then the associated transform

$$
F\left(s_{1}, s_{2}, \ldots, s_{n}\right) \stackrel{A_{n}}{\longrightarrow} G(s)=\frac{k}{a}\left[G_{1}(s)-G_{1}(s+a)\right]
$$

where $A_{n}$ means the association process for finding $G(s)$ from $F\left(s_{1}, s_{2}, \ldots, s_{n}\right)$. $A_{n-1}$ has the similar meaning.

Proof: By Equations (1.1) and (1.2), we have

$$
\begin{aligned}
& g(t)=\left.f\left(t_{1}, t_{2}, \ldots, t_{n}\right)\right|_{t_{1}=t_{2}=\ldots=t_{n}=t} \\
& =L_{n}^{-1}\left[F\left(s_{1}, s_{2}, \ldots, s_{n}\right) ; t_{1}, t_{2}, \ldots, t_{n}\right]_{t_{1}=t_{2}}=\ldots t_{n}=t \\
& =\frac{1}{(2 \pi i)^{n}}{ }_{\alpha_{1}-i \infty}^{\alpha_{1}+i \infty} \int_{\alpha_{2}-i \infty}^{\alpha_{2}+i \infty} \ldots \int_{\alpha_{n}-i \infty}^{\alpha_{n}^{+i \infty}} F\left(s_{1}, s_{2}, \ldots, s_{n}\right) \\
& \cdot \exp \left(\sum_{j=1}^{n} s_{j} t\right) d s_{1} d s_{2} \ldots d s_{n} \\
& =\frac{1}{(2 \pi i)^{n}} \int_{\alpha_{1}-i \infty}^{\alpha_{1}+j \infty} \ldots \int_{\alpha_{n}-i \infty}^{\alpha_{n}+i \infty} \frac{k}{s_{m}\left(s_{m}+a\right)} F_{1}\left(s_{1}, \ldots, s_{m-1}, s_{m+1}, \ldots, s_{1}\right. \\
& \cdot \exp \left(\sum_{j=1}^{n} s_{j} t\right) d s_{1} d s_{2} \ldots d s_{n}
\end{aligned}
$$




$$
\begin{aligned}
& =\frac{1}{2 \pi i} \int_{\alpha_{m}-i \infty}^{\alpha_{m}+i \infty} \frac{k}{s_{m}\left(s_{m}+a\right)} \exp \left(s_{m} t\right) d s_{m} . \\
& \frac{1}{(2 \pi i)^{n-1}} \int_{\alpha_{1}-i_{\infty}}^{\alpha_{1}+i_{\infty}} \cdots \alpha_{m-1}^{\alpha_{m-1}+i_{\infty}} \alpha_{m+1}^{\alpha_{m+1}} \alpha^{-i \infty} \cdots \alpha_{n} \alpha^{-i \infty} \\
& F\left(s_{1}, \ldots, s_{m-1}, s_{m+1}, \ldots, s_{n}\right) \exp \left(\sum_{\substack{j=1 \\
j \neq m}}^{n} s_{j} t\right) d s_{1} \ldots d s_{m-1} d s_{m+1} \ldots d s_{n} \\
& =k L_{1}^{-1}\left[\frac{1}{s_{m}\left(s_{m}+a\right)} ; t\right] L_{n-1}^{-1}\left[F_{1}\left(s_{1}, \ldots, s_{m-1}, s_{m+1}, \ldots, s_{n}\right) ; t, t, \ldots, t\right]
\end{aligned}
$$

Using the table of inverse Laplace transform given in [8], we get

$$
\begin{aligned}
g(t) & =k \frac{1-\exp (-a t)}{a} g_{1}(t) \\
& =\frac{k}{a}\left[g_{1}(t)-\exp (-a t) g_{1}(t)\right]
\end{aligned}
$$

Taking Laplace transform of both sides of the Equation (2.1) yields

$$
G(s)=\frac{k}{a}\left[G_{1}(s)-G_{1}(s+a)\right]
$$

Hence the theorem is proved.

Example 2.1

Consider

$$
F\left(s_{1}, s_{2}, s_{3}\right)=\frac{k}{s_{3}\left(s_{1}+a\right)\left(s_{2}+b\right)\left(s_{3}+c\right)}
$$

and let

$$
F_{1}\left(s_{1}, s_{2}\right)=\frac{1}{\left(s_{1}+a\right)\left(s_{2}+b\right)}
$$

Using the table given in [6]

$$
F_{1}\left(s_{1}, s_{2}\right) \stackrel{A_{2}}{\longrightarrow} G_{1}(s)=\frac{1}{s+a+b}
$$

By Theorem 2.1,

\section{Example 2.2}

$$
\begin{aligned}
F\left(s_{1}, s_{2}, s_{3}\right) \stackrel{A_{3}}{\rightarrow} G(s)=\frac{k}{c}\left[\frac{1}{s+a+b}-\frac{1}{s+a+b+c}\right] \\
=\frac{k}{(s+a+b)(s+a+b+c)}
\end{aligned}
$$

Let

$$
F\left(s_{1}, s_{2}, s_{3}\right)=\frac{k}{\left[a\left(s_{1}+s_{2}\right)^{2}+b\left(s_{1}+s_{2}\right)+c\right] s_{3}\left(s_{3}+d\right)}
$$


and

$$
F_{1}\left(s_{1}, s_{2}\right)=\frac{1}{a\left(s_{1}+s_{2}\right)^{2}+b\left(s_{1}+s_{2}\right)+c}
$$

Use of the table given in [6] gives

$$
F_{1}\left(s_{1}, s_{2}\right) \stackrel{A_{2}}{\longrightarrow} G_{1}(s)=\frac{1}{a s^{2}+b s+c}
$$

Theorem 2.1 yields

$$
\begin{aligned}
F\left(s_{1}, s_{2}, s_{3}\right) \stackrel{A_{3}}{\longrightarrow} G(s) & =\frac{k}{d}\left[\frac{1}{a s^{2}+b s+c}-\frac{1}{a(s+d)^{2}+b(s+d)+c}\right] \\
& =\frac{k(2 a s+a d+b)}{\left(a s^{2}+b s+c\right)\left\{a(s+d)^{2}+b(s+d)+c\right\}}
\end{aligned}
$$

Theorem 2.2. If a given function $F\left(s_{1}, s_{2}, \ldots, s_{n}\right)$ can be factored in the form

$$
F\left(s_{1}, s_{2}, \ldots, s_{n}\right)=\frac{k\left(s_{m}+a\right)}{\left(s_{m}+\alpha\right)\left(s_{m}+\beta\right)} F_{1}\left(s_{1}, \ldots, s_{m-1}, s_{m+1}, \ldots, s_{n}\right)
$$

and if $F_{1}\left(s_{1}, s_{2}, \ldots, s_{m-1}, s_{m+1}, \ldots, s_{n}\right) \stackrel{A_{n-1}}{\rightarrow} G_{1}(s)$. Then the associated transform

$$
F\left(s_{1}, s_{2}, \ldots, s_{n}\right) \stackrel{A_{n}}{\longrightarrow} G(s)=\frac{\alpha-a}{\alpha-\beta} G_{1}(s+\alpha)+\frac{\beta-a}{\beta-\alpha} G_{1}(s+\beta)
$$

Proof. By Equations (1.1) and (1.2), we get

$$
\begin{aligned}
& g(t)=\left.f\left(t_{1}, t_{2}, \ldots, t_{n}\right)\right|_{t_{1}=t_{2}=\ldots=t_{n}=t} \\
& =\left.L_{n}^{-1}\left[F\left(s_{1}, s_{2}, \ldots, s_{n}\right) ; t_{1}, t_{2}, \ldots, t_{n}\right]\right|_{t_{1}=t_{2}=\ldots=t_{n}=t} \\
& =\frac{1}{(2 \pi i)^{n}}{ }_{\alpha_{1}-i^{\infty}}^{\alpha_{1}+j_{\infty}} \ldots \int_{\alpha_{n}-i_{\infty}}^{\alpha_{n}+i_{\infty}} F\left(s_{1}, s_{2}, \ldots, s_{n}\right) \\
& \cdot \exp \left(\sum_{j=1}^{n} s_{j} t\right) d s_{1} \ldots d s_{n} \\
& =\frac{1}{(2 \pi i)^{n}}{ }_{\alpha_{1}-i \infty}^{\alpha_{1}+i_{\infty}} \cdots \int_{\alpha_{n}-i_{\infty}}^{\alpha_{n}+j_{\infty}} \frac{k\left(s_{m}+a\right)}{\left(s_{m}+\alpha\right)\left(s_{m}+\beta\right)} F_{1}\left(s_{1}, \ldots, s_{m-1}, s_{m+1}, \ldots, s_{,},\right. \\
& \cdot \exp \left(\sum_{j=1}^{n} s_{j} t\right) d s_{1} d s_{2} \ldots d s_{n}
\end{aligned}
$$




$$
\begin{aligned}
& =\frac{1}{2 \pi i} \int_{\alpha_{m}-i \infty}^{\alpha_{m}^{+i \infty}} \frac{k\left(s_{m}+a\right)}{\left(s_{m}+\alpha\right)\left(s_{m}+\beta\right)} \exp \left(s_{m} t\right) d s_{m}
\end{aligned}
$$

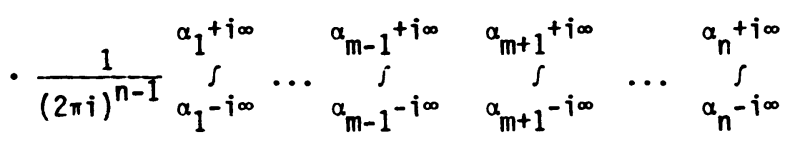

$$
\begin{aligned}
& F_{1}\left(s_{1}, \ldots, s_{m-1}, s_{m+1}, \ldots, s_{n}\right) \exp \left(\sum_{\substack{j=1 \\
j \neq m}}^{n} s_{j} t\right) d s_{1} \ldots d s_{m-1} d s_{m+1} \ldots d s_{n} \\
& =k L_{1}^{-1}\left[\frac{\left(s_{m}+a\right)}{\left(s_{m}+\alpha\right)\left(s_{m}+B\right)} ; t\right] L_{n-1}^{-1}\left[F_{1}\left(s_{1}, \ldots, s_{m-1}, s_{m+1}, \ldots, s_{n}\right) ; t, t, \ldots, t\right]
\end{aligned}
$$

Referring to the table of inverse Laplace transform [8],

$$
\begin{aligned}
g(t) & =k\left[\frac{\alpha-a}{\alpha-\beta} \exp (-\alpha t)+\frac{\beta-a}{\beta-\alpha} \exp (-\beta t)\right] g_{1}(t) \\
& =\frac{k(\alpha-a)}{\alpha-\beta} \exp (-\alpha t) g_{1}(t)+\frac{k(\beta-a)}{\beta-\alpha} \exp (-\beta t) g_{1}(t)
\end{aligned}
$$

On taking Laplace transform of both sides of Equation (2.2), we obtain

$$
G(s)=\frac{k(\alpha-a)}{\alpha-\beta} G_{1}(s+\alpha)+\frac{k(\beta-a)}{\beta-\alpha} G_{1}(s+\beta)
$$

This establishes the theorem.

\section{Example 2.3}

Suppose

$$
F\left(s_{1}, s_{2}, s_{3}\right)=\frac{k\left(s_{3}+c\right)}{\left(s_{1}+a\right)\left(s_{2}+b\right)\left(s_{3}+\alpha\right)\left(s_{3}+\beta\right)}
$$

and let

$$
F_{1}\left(s_{1}, s_{2}\right)=\frac{1}{\left(s_{1}+a\right)\left(s_{2}+b\right)}
$$

From the table shown in [6],

$$
F_{1}\left(s_{1}, s_{2}\right) \stackrel{A_{2}}{\longrightarrow} G_{1}(s)=\frac{1}{(s+a+b)}
$$

Then by using Theorem 2.2, we get

$$
\begin{aligned}
F\left(s_{1}, s_{2}, s_{3}\right) \stackrel{A_{3}}{\longrightarrow} G(s) & =\left(\frac{\alpha-c}{\alpha-\beta}\right)\left(\frac{k}{s+a+a+b}\right)+\left(\frac{\beta-c}{\beta-\alpha}\right)\left(\frac{k}{s+\beta+a+b}\right) \\
& =\frac{k}{\alpha-\beta}\left[\frac{\alpha-c}{s+a+a+b}-\frac{\beta-c}{s+\beta+a+b}\right] \\
& =\frac{k(s+a+b+c)}{(s+\alpha+a+b)(s+\beta+a+b)}
\end{aligned}
$$




\section{Example 2.4}

Consider

$$
F\left(s_{1}, s_{2}, s_{2}\right)=\frac{k\left(s_{3}+d\right)}{\left[a\left(s_{1}+s_{2}\right)^{2}+b\left(s_{1}+s_{2}\right)+c\right]\left(s_{3}+\alpha\right)\left(s_{3}+\beta\right)}
$$

and suppose

$$
F_{1}\left(s_{1}, s_{2}\right) \fallingdotseq \frac{1}{a\left(s_{1}+s_{2}\right)^{2}+b\left(s_{1}+s_{2}\right)+c}
$$

Using the table shown in $[\epsilon]$, we cbtain

$$
F_{1}\left(s_{1}, s_{2}\right) \stackrel{A_{1}}{\rightarrow} G_{1}(s)=\frac{1}{a s^{2}+b s+c}
$$

Then Theorem 2.2 gives the associated transform

$$
\begin{aligned}
F\left(s_{1}, s_{2}, s_{3}\right) \stackrel{A_{3}}{\longrightarrow} G(s)= & k\left(\frac{\alpha-d}{\alpha-\beta}\right)\left(\frac{1}{a(s+\alpha)^{2}+b(s+\alpha)+c}\right)+k\left(\frac{\beta-d}{\beta-\alpha}\right) D\left(\frac{1}{a(s+\beta)^{2}+b(s+\beta)+c}\right) \\
& -\frac{k}{\alpha-\beta}\left[\frac{(\alpha-d)}{a(s+\alpha)^{2}+b(s+\alpha)+c}+\frac{(d-\beta)}{a(s+\beta)^{2}+b(s+\beta)+c}\right] \\
= & \frac{k d[2 a s+a(\alpha+\beta)+b]+a s^{2}+b s-a \alpha \beta}{\left\{a(s+\alpha)^{2}+b(s+\alpha)+c\right\}\left\{a(s+\beta)^{2}+b(s+\beta)+c\right\}}
\end{aligned}
$$

Theorem 2.3. If a function $F\left(s_{1}, s_{2}, \ldots, s_{n}\right)$ is of the form

$$
F\left(s_{1}, s_{2}, \ldots, s_{n}\right)=\frac{k}{s_{m}\left(s_{m}+\alpha\right)^{2}} F_{1}\left(s_{1}, s_{2}, \ldots, s_{m-1}, s_{m+1}, \ldots, s_{n}\right)
$$

with

$$
F_{1}\left(s_{1}, \ldots, s_{m-1}, s_{m+1}, \ldots, s_{n}\right) \stackrel{A_{n-1}}{\longrightarrow} G_{1}(s)
$$

Then

$$
F\left(s_{1}, s_{2}, \ldots, s_{n}\right) \stackrel{A_{n}}{\longrightarrow} G(s)=\frac{k}{\alpha^{2}} G_{1}(s)-\frac{k}{\alpha}\left[\frac{1}{\alpha} G_{1}(s+\alpha)+(-1) \frac{d}{d s} G_{1}(s+\alpha)\right]
$$

Proof: By definitions (1.1) and (1.2)

$$
\begin{aligned}
g(t) & =\left.f\left(t_{1}, t_{2}, \ldots, t_{n}\right)\right|_{t_{1}=t_{2}=\ldots=t_{n}=t} \\
& =L_{n}^{-1}\left[F\left(s_{1}, s_{2}, \ldots, s_{n}\right) ; t, t, \ldots, t\right]
\end{aligned}
$$




$$
\begin{aligned}
& =\frac{1}{2 \pi i} \int_{\alpha_{m}-i \infty}^{\alpha_{m}^{+i \infty}} \frac{k}{s_{m}\left(s_{m}+\alpha\right)^{2}} \exp \left(s_{m} t\right) d s_{m}
\end{aligned}
$$

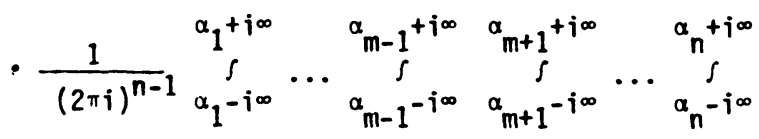

$$
\begin{aligned}
& F_{1}\left(s_{1}, \ldots, s_{m-1}, s_{m+1}, \ldots, s_{n}\right) \exp \left(\sum_{\substack{j=1 \\
j \neq m}}^{n} s_{j} t\right) d s_{1} \ldots d s_{m-1} d s_{m+1} \ldots d s_{n}
\end{aligned}
$$

Using the results of inverse Laplace transform from [8], we get

$$
\begin{aligned}
g(t) & =k\left[\frac{1}{\alpha^{2}}-\frac{1}{\alpha}\left(\frac{1}{\alpha}+t\right) \exp (-\alpha t)\right] g_{1}(t) \\
& =\frac{k}{\alpha^{2}} g_{1}(t)-\frac{k}{\alpha^{2}} \exp (-\alpha t) g_{1}(t)-\frac{k}{\alpha} t \exp (-\alpha t) g(t)
\end{aligned}
$$

Taking Laplace transform on both sides of (2.3),

$$
G(s)=\frac{k}{\alpha^{2}} G_{1}(s)-\frac{k}{\alpha}\left[\frac{1}{\alpha} G_{1}(s+\alpha)+(-1) \frac{d}{d s} G_{1}(s+\alpha)\right] \text {. }
$$

Example 2.5

Consider

$$
F\left(s_{1}, s_{2}, s_{3}\right)=\frac{k}{\left(s_{1}+a\right)\left(s_{2}+b\right) s_{3}\left(s_{3}+\alpha\right)^{2}}
$$

Direct use of the table given in [6], we find

$$
F_{1}\left(s_{1}, s_{2}\right)=\frac{1}{\left(s_{1}+a\right)\left(s_{2}+b\right)} \stackrel{A_{2}}{\longrightarrow} G_{1}(s)=\frac{1}{s+a+b}
$$

Thus, by Theorem 2.3,

$$
F\left(s_{1}, s_{2}, s_{3}\right) \stackrel{A_{3}}{\longrightarrow} G(s)=\frac{k}{a^{2}(s+a+b)}-\frac{k}{\alpha}\left[\frac{1}{a(s+a+a+b)}+\frac{1}{(s+a+a+b)^{2}}\right]
$$

or

\section{Example 2.6}

$$
G(s)=\frac{k}{\alpha}\left[\frac{1}{\alpha(s+a+b)}-\frac{s+2 \alpha+a+b}{\alpha(s+\alpha+a+b)^{2}}\right]
$$

\section{Suppose}

$$
F\left(s_{1}, s_{2}, s_{3}\right)=\frac{k}{\left[s_{3}\left(s_{3}+\alpha\right)^{2}\right]\left[a\left(s_{1}+s_{2}\right)^{2}+b\left(s_{1}+s_{2}\right)+c\right]}
$$


Use of the results of [6],

$$
F_{1}\left(s_{1}, s_{2}\right)=\frac{1}{a\left(s_{1}+s_{2}\right)^{2}+b\left(s_{1}+s_{2}\right)+c} \stackrel{A_{2}}{\longrightarrow} G_{1}(s)=\frac{1}{a s^{2}+b s+c}
$$

Then Theorem 2.3 yields

$$
F\left(s_{1}, s_{2}, s_{3}\right) \stackrel{A_{3}}{\longrightarrow} G(s)=\frac{k}{\alpha^{2}}\left(\frac{1}{a s^{2}+b s+c}\right)-\frac{k}{\alpha}\left[\frac{1}{\alpha\left\{a(s+\alpha)^{2}+b(s+a)+c\right\}}+\frac{2 a(s+\alpha)+b}{\left\{a(s+\alpha)^{2}+b(s+\alpha)+c\right\}^{2}}\right]
$$

Theorem 2.4. If $F\left(s_{1}, s_{2}, \ldots, s_{n}\right)$ can be expressed in the following form

$$
F\left(s_{1}, s_{2}, \ldots, s_{n}\right)=\frac{k\left(s_{m}+a\right)}{s_{m}\left(s_{m}^{2}-\alpha^{2}\right)} F_{1}\left(s_{1}, \ldots, s_{m-1}, s_{m+1}, \ldots, s_{n}\right)
$$

where

$$
F_{1}\left(s_{1}, \ldots, s_{m-1}, s_{m+1}, \ldots, s_{n}\right) \stackrel{A_{n-1}}{\longrightarrow} G_{1}(s)
$$

Then the associated transform

$$
G(s)=\frac{k(a+\alpha)}{2 \alpha^{2}} G_{1}(s-\alpha)+\frac{k(a-\alpha)}{2 \alpha^{2}} G_{1}(s+\alpha)-\frac{k a}{\alpha^{2}} G_{1}(s)
$$

Proof: By definitions (1.1) and (1.2),

$$
\begin{aligned}
& g(t)=\left.L_{n}^{-1}\left[F\left(s_{1}, s_{2}, \ldots, s_{n}\right) ; t_{1}, t_{2}, \ldots, t_{n}\right]\right|_{t_{1}=t_{2}=\ldots=t_{n}=t} \\
& =\frac{1}{2 \pi i} \int_{\alpha_{m}-i_{\infty}}^{\alpha_{m}^{+i \infty}} \frac{\left(s_{m}+a\right) k}{s_{m}\left(s_{m}^{2}-\alpha^{2}\right)} \exp \left(s_{m} t\right) d s_{m} \\
& \frac{1}{(2 \pi i)^{n-1}} \int_{\alpha_{1}-i_{\infty}}^{\alpha_{1}+i_{\infty}} \cdots \int_{a_{m-1}-i_{\infty}}^{\alpha_{m-1}+i_{\infty}} \int_{\alpha_{m+1}-i_{\infty}}^{\alpha_{m+1}+i_{\infty}} \cdots \int_{\alpha_{n}-i_{\infty}}^{\alpha_{n}+i_{\infty}} F\left(s_{1}, \ldots, s_{m-1}, s_{m+1}, \ldots, s_{n}\right) \\
& \cdot \exp \left(\sum_{\substack{j=1 \\
j \neq m}}^{n} s_{j} t\right) d s_{1} \ldots d s_{m-1} d s_{m+1} \ldots d s_{n}
\end{aligned}
$$

By the results of inverse Laplace transform shown in [8], we obtain

$$
g(t)=\left[\frac{k}{\alpha} \sinh (\alpha t)+\frac{k a}{2} \cosh (\alpha t)-\frac{k a}{\alpha}\right] g_{1}(t)
$$

On taking Laplace transform of both sides of (2.4),

$$
L[g(t) ; s]=L\left[\frac{k}{\alpha} \sinh (\alpha t) g_{1}(t)+\frac{k a}{\alpha^{2}} \cosh (\alpha t) g_{1}(t)-\frac{k a}{\alpha^{2}} g_{1}(t) ; s\right]
$$




$$
G(s)=\frac{k}{2 \alpha}\left[G_{1}(s-\alpha)-G_{1}(s+\alpha)\right]+\frac{k a}{2 \alpha^{2}}\left[G_{1}(s-\alpha)+G_{1}(s+\alpha)\right]-\frac{k a}{\alpha^{2}} G_{1}(s)
$$

or

$$
G(s)=\frac{k(a+\alpha)}{2 \alpha^{2}} G_{1}(s-\alpha)+\frac{k(a-\alpha)}{2 \alpha^{2}} G_{1}(s+\alpha)-\frac{k a}{\alpha^{2}} G_{1}(s) .
$$

\section{Example 2.7}

Let

$$
F\left(s_{1}, s_{2}, s_{3}\right)=\frac{k\left(s_{3}+c\right)}{s_{3}\left(s_{3}^{2}-a^{2}\right)\left(s_{1}+a\right)\left(s_{2}+b\right)}
$$

Then

$$
F_{1}\left(s_{1}, s_{2}\right)=\frac{1}{\left(s_{1}+a\right)\left(s_{2}+b\right)} \stackrel{A_{2}}{\longrightarrow} G_{1}(s)=\frac{1}{s+a+b}
$$

and by applying Theorem 2.4,

$$
\begin{aligned}
F\left(s_{1}, s_{2}, s_{3}\right) \stackrel{A_{3}}{\longrightarrow} G(s) & =\frac{k}{2 \alpha}\left[\frac{1}{s-a+a+b}-\frac{1}{s+a+a+b}\right]+\frac{c k}{2 \alpha^{2}}\left[\frac{1}{s-a+a+b}+\frac{1}{s+a+a+b}\right]-\frac{c k}{\alpha^{2}} \frac{1}{s+a+b} \\
& =\frac{k(c+a)}{2 \alpha^{2}(s-a+a+b)}+\frac{k(c-a)}{2 \alpha^{2}(s+a+a+b)}-\frac{c k}{\alpha^{2}(s+a+b)} .
\end{aligned}
$$

\section{Example 2.8}

Considering

$$
F\left(s_{1}, s_{2}, s_{3}\right)=\frac{\left(s_{3}+d\right) k}{s_{3}\left(s_{3}^{2}-a^{2}\right)\left[a\left(s_{1}+s_{2}\right)^{2}+b\left(s_{1}+s_{2}\right)+c\right]}
$$

we find

$$
F_{1}\left(s_{1}, s_{2}\right)=\frac{1}{a\left(s_{1}+s_{2}\right)^{2}+b\left(s_{1}+s_{2}\right)+c} \stackrel{A_{2}}{\longrightarrow} G_{1}(s)=\frac{1}{a s^{2}+b s+c}
$$

and Theorem 2.4 shows that

$$
\begin{aligned}
F\left(s_{1}, s_{2}, s_{3}\right) \stackrel{A_{3}}{\longrightarrow} G(s)= & \frac{k(d+\alpha)}{2 \alpha^{2}\left\{a(s-\alpha)^{2}+b(s-\alpha)+c\right\}} \\
& +\frac{k(d-\alpha)}{2 \alpha^{2}\left\{a(s+\alpha)^{2}+b(s+\alpha)+c\right\}} \\
& +\frac{k d}{\alpha^{2}\left(a s^{2}+b s+c\right)} .
\end{aligned}
$$


Theorem 2.5. If a function $F\left(s_{1}, s_{2}, \ldots, s_{n}\right)$ can be factored in the form

$$
F\left(s_{1}, s_{2}, \ldots, s_{n}\right)=\frac{k}{s_{m}\left(s_{m}^{3}+\alpha^{3}\right)} F_{1}\left(s_{1}, \ldots, s_{m-1}, s_{m+1}, \ldots, s_{n}\right)
$$

Then

$$
\begin{aligned}
F\left(s_{1}, s_{2}, \ldots, s_{n}\right) \stackrel{A_{n}}{\longrightarrow} G(s)= & \frac{k}{3 \alpha^{3}}\left[3 G_{1}(s)-G_{1}(s+\alpha)-\right. \\
& \left.G_{1}\left(s-\frac{\alpha}{2}-\frac{\sqrt{3}}{2} i_{\alpha}\right)-G_{1}\left(s-\frac{\alpha}{2}+\frac{\sqrt{3}}{2} i_{\alpha}\right)\right]
\end{aligned}
$$

where $F_{1}\left(s_{1}, \ldots, s_{m-1}, s_{m+1}, \ldots, s_{n}\right) \stackrel{A_{n-1}}{\longrightarrow} G_{1}(s)$.

Proof: By definitions (1.1) and (1.2)

$$
\begin{aligned}
g(t) & =L_{n}^{-1}\left[F\left(s_{1}, s_{2}, \ldots, s_{n}\right) ; t, t, \ldots, t\right] \\
& =k \cdot L_{1}^{-1}\left[\frac{1}{s_{m}\left(s_{m}^{3}+\alpha\right.} ; t\right] \cdot L_{n-1}^{-1}\left[F_{1}\left(s_{1}, \ldots, s_{m-1}, s_{m+1}, \ldots, s_{n}\right) ; t, t, \ldots, t\right]
\end{aligned}
$$

Referring to the results given in [8],

$$
g(t)=k \cdot \frac{1}{\alpha^{3}}\left[1-\frac{1}{3} \exp (-\alpha t)-\frac{2}{3} \exp \left(\frac{\alpha t}{2}\right) \cos \left(\frac{\sqrt{3}}{2} \alpha t\right)\right] g_{1}(t)
$$

Taking Laplace transform on both sides,

$$
L[g(t) ; s]=\frac{k}{\alpha} L\left[g_{1}(t)-\frac{1}{3} \exp (-\alpha t) g_{1}(t)-\frac{2}{3} \exp \left(\frac{\alpha t}{2}\right) \cos \left(\frac{\sqrt{3}}{2} \alpha t\right) g_{1}(t) ; s\right],
$$

we obtain

$$
G(s)=\frac{k}{3_{\alpha}{ }^{3}}\left[3 G_{1}(s)-G_{1}(s+\alpha)-G_{1}\left(s-\frac{\alpha}{2}-\frac{\sqrt{3}}{2} i_{\alpha}\right)-G_{1}\left(s-\frac{\alpha}{2}+\frac{\sqrt{3}}{2} i_{\alpha}\right)\right] .
$$

Example 2.9

Suppose

$$
F\left(s_{1}, s_{2}, s_{3}\right)=\frac{k}{\left(s_{1}+a\right)\left(s_{2}+b\right) s_{3}\left(s_{3}^{3}+{ }^{3}\right)}
$$

Then

$$
F_{1}\left(s_{1}, s_{2}\right)=\frac{1}{\left(s_{1}+a\right)\left(s_{2}+b\right)} \stackrel{A_{2}}{-} \cdot G_{1}(s)=\frac{1}{s+a+b}
$$

So, by Theorem 2.5 ,

$$
F\left(s_{1}, s_{2}, s_{3}\right) \stackrel{A_{3}}{\longrightarrow} G(s)=\frac{k}{3 \alpha^{3}}\left[\frac{3}{s+a+b}-\frac{1}{s+a+a+b}-\frac{1}{s-\frac{\alpha}{2}(1+\sqrt{3} i)+a+b}-\frac{1}{s-\frac{a}{2}(1-\sqrt{3} i)+a+b}\right]
$$


Example 2.10

Consider

$$
F\left(s_{1}, s_{2}, s_{3}\right)=\frac{k}{s_{3}\left(s_{3}^{3}+\alpha^{3}\right)\left\{a\left(s_{1}+s_{2}\right)^{2}+b\left(s_{1}+s_{2}\right)+c\right\}}
$$

Then

$$
F_{1}\left(s_{1}, s_{2}\right)=\frac{1}{a\left(s_{1}+s_{2}\right)^{2}+b\left(s_{1}+s_{2}\right)+c} \stackrel{A_{2}}{\longrightarrow} G_{1}(s)=\frac{1}{a s^{2}+b s+c}
$$

By applying Theorem 2.5, we find

$$
\begin{aligned}
F\left(s_{1}, s_{2}, s_{3}\right) \stackrel{A_{3}}{\longrightarrow} G(s)= & \frac{k}{3 \alpha^{3}}\left[\frac{3}{a s^{2}+b s+c}-\frac{1}{a(s+a)^{2}+b(s+a)+c}\right. \\
& -\frac{1}{a\left\{s-\frac{\alpha}{2}(1+\sqrt{3} i)\right\}^{2}+b\left\{s-\frac{\alpha}{2}(1+\sqrt{3} i)\right\}+c} \\
& \left.-\frac{1}{a\left\{s-\frac{\alpha}{2}(1-\sqrt{3} i)\right\}^{2}+b\left\{s-\frac{\alpha}{2}(1-\sqrt{3} i)\right\}+c}\right]
\end{aligned}
$$

Theorem 2.6.

If a function

$$
F\left(s_{1}, s_{2}, \ldots, s_{n}\right)=\frac{k}{s_{m}^{2}\left(s_{m}+\alpha\right)} F_{1}\left(s_{1}, \ldots, s_{m-1}, s_{m+1}, \ldots, s_{n}\right)
$$

then its associated transform

$$
G(s)=\frac{k}{\alpha^{2}}\left[G_{1}(s+\alpha)-\alpha \frac{d}{d s} G_{1}(s)-G_{1}(s)\right]
$$

Proof. By definitions

$$
\begin{aligned}
g(t) & =L_{n}^{-1}\left[F\left(s_{1}, s_{2}, \ldots, s_{n}\right) ; t, t, \ldots t\right] \\
& =k L_{1}^{-1}\left[\frac{1}{s_{m}^{2}\left(s_{m}+\alpha\right)} ; t\right] \cdot L_{n-1}^{-1}\left[F_{1}\left(s_{1}, \ldots, s_{m-1}, s_{m+1}, \ldots, s_{n} ; t, t, \ldots, t\right]\right. \\
& =\frac{k}{\alpha}[\exp (-\alpha t)+\alpha t-1] g_{1}(t)
\end{aligned}
$$

On taking Laplace transform on both sides, one obtains

$$
\begin{aligned}
G(s) & =\frac{k}{\alpha^{2}} L\left[\exp (-\alpha t) g_{1}(t)+\alpha \operatorname{tg} g_{1}(t)-g_{1}(t) ; s\right] \\
& =\frac{k}{\alpha^{2}}\left[G_{1}(s+\alpha)-\alpha \frac{d}{d s} G_{1}(s)-G_{1}(s)\right]
\end{aligned}
$$




\section{Example 2.11}

Take

$$
F\left(s_{1}, s_{2}, s_{3}\right)=\frac{k}{\left(s_{1}+a\right)\left(s_{2}+b\right) s_{3}^{2}\left(s_{3}+\alpha\right)}
$$

Thus as before

$$
F_{1}\left(s_{1}, s_{2}\right) \stackrel{A_{2}}{\longrightarrow} G_{1}(s)=\frac{1}{s+a+b}
$$

and by Theorem 2.6

$$
F\left(s_{1}, s_{2}, s_{3}\right) \stackrel{A_{3}}{\longrightarrow} G(s)=\frac{k}{a^{2}}\left[\frac{(s+a+b)^{2}+a-s(a+b+c)}{(s+a+a+b)(s+a+b)^{2}}\right]
$$

\section{Example 2.12}

Let

$$
F\left(s_{1}, s_{2}, s_{3}\right)=\frac{k}{\left\{a\left(s_{1}+s_{2}\right)^{2}+b\left(s_{1}+s_{2}\right)+c\right\} s_{3}^{2}\left(s_{3}+\alpha\right)}
$$

Thus as before

$$
F_{1}\left(s_{1}, s_{2}\right) \stackrel{A_{2}}{\longrightarrow} G_{1}(s)=\frac{1}{a s^{2}+b s+c}
$$

and by Theorem 2.6

$$
F\left(s_{1}, s_{2}, s_{3}\right) \stackrel{A_{3}}{\longrightarrow} G(s)=\frac{k}{\alpha^{2}}\left[\frac{1}{a(s+a)^{2}+b(s+a)+c}+\frac{\alpha(2 a s+b)}{\left(a s^{2}+b s+c\right)^{2}}-\frac{1}{a s^{2}+b s+c}\right]
$$

Following analogous arguments, it is easy to prove the following results.

Theorem 2.7.

If a function

$$
F\left(s_{1}, s_{2}, \ldots, s_{n}\right)=\frac{k\left(s_{m}+a\right)}{s_{m}^{2}\left(s_{m}+\alpha\right)} F_{1}\left(s_{1}, \ldots, s_{m-1}, s_{m+1}, \ldots, s_{n}\right),
$$

then its associated transform

$$
G(s)=k\left(\frac{1}{\alpha}-\frac{a}{\alpha^{2}}\right)\left[G_{1}(s)-G_{1}(s+\alpha)\right]-\frac{a k}{\alpha} \frac{d}{d s} G_{1}(s)
$$

Example 2.13

Consider

$$
F_{1}\left(s_{1}, s_{2}, s_{3}\right)=\frac{\left(s_{3}+c\right) k}{\left(s_{1}+a\right)\left(s_{2}+b\right) s_{3}^{2}\left(s_{3}+\alpha\right)}
$$

and

$$
F_{1}\left(s_{1}, s_{2}\right)=\frac{1}{\left.\left(s_{1}+a\right) s_{2}+b\right)}
$$


Then using Theorem 2.7, we can get.

$$
G(s)=\frac{k(s+a+b+c)}{(s+a+b)^{2}(s+a+a+b)}
$$

\section{Example 2.14}

Considering

$$
F_{1}\left(s_{1}, s_{2}, s_{3}\right)=\frac{\left(s_{3}+c\right) k}{s_{3}^{2}\left(s_{3}+\alpha\right)\left\{a\left(s_{1}+s_{2}\right)^{2}+b\left(s_{1}+s_{2}\right)+c\right\}}
$$

and

$$
F_{1}\left(s_{1}, s_{2}\right)=\frac{1}{a\left(s_{1}+s_{2}\right)^{2}+b\left(s_{1}+s_{2}\right)+c}
$$

we obtain, by Theorem 2.7,

$$
G(s)=\frac{k}{\alpha^{2}}(a-c)\left[\frac{1}{a s^{2}+b s+c}-\frac{1}{a(s+a)^{2}+b(s+a)+c}\right]+\frac{c k(2 a s+b)}{\alpha\left(a s^{2}+b s+c\right)} .
$$

Theorem 2.8.

If a function

$$
F\left(s_{1}, s_{2}, \ldots, s_{n}\right)=\frac{k}{\left(s_{m}+\alpha\right)\left(s_{m}+\beta\right)\left(s_{m}+\gamma\right)} F_{1}\left(s_{1}, \ldots, s_{m-1}, s_{m+1}, \ldots, s_{n}\right),
$$

then its associated transform is given by

$$
G(s)=k\left[\frac{G_{1}(s+\alpha)}{(\beta-\alpha)(\gamma-\alpha)}+\frac{G_{1}(s+\beta)}{(\alpha-\beta)(\gamma-\beta)}+\frac{G_{1}(s+\gamma)}{(\alpha-\gamma)(\beta-\gamma)}\right]
$$

\section{Example 2.15}

Let

$$
F_{1}\left(s_{1}, s_{2}, s_{3}\right)=\frac{k\left(s_{3}+\alpha\right)^{-1}\left(s_{3}+\beta\right)^{-1}\left(s_{3}+\gamma\right)^{-1}}{\left(s_{1}+a\right)\left(s_{2}+b\right)}
$$

and

$$
F\left(s_{1}, s_{2}\right)=\frac{1}{\left(s_{1}+a\right)\left(s_{2}+b\right)}
$$

Then the use of Theorem 2.8 yields

$$
G(s)=k\left[\frac{(\beta-\alpha)^{-1}(\gamma-\alpha)^{-1}}{s+\alpha+a+b}+\frac{(\alpha-\beta)^{-1}(\gamma-\beta)^{-1}}{s+\beta+a+b}+\frac{(\alpha-\gamma)^{-1}(\beta-\gamma)^{-1}}{s+\gamma+a+b}\right]
$$

\section{Example 2.16}

Suppose

$$
F\left(s_{1}, s_{2}, s_{3}\right)=\frac{k\left(s_{3}+\alpha\right)^{-1}\left(s_{3}+\beta\right)^{-1}\left(s_{3}+\gamma\right)^{-1}}{a\left(s_{1}+s_{2}\right)^{2}+b\left(s_{1}+s_{2}\right)+c}
$$


and take

$$
F_{1}\left(s_{1}, s_{2}\right)=\frac{1}{a\left(s_{1}+s_{2}\right)^{2}+b\left(s_{1}+s_{2}\right)+c}
$$

Then, direct application of Theorem 2.8 gives,

$$
G(s)=k\left[\frac{(\beta-\alpha)^{-1}(\gamma-\alpha)^{-1}}{a(s+\alpha)^{2}+b(s+\alpha)+c}+\frac{(\alpha-\beta)^{-1}(\gamma-\beta)^{-1}}{a(s+\beta)^{2}+b(s+\beta)+c}+\frac{(\alpha-\gamma)^{-1}(\beta-\gamma)^{-1}}{a(s+\gamma)^{2}+b(s+\gamma)+c}\right] .
$$

3. CONCLUSIONS.

Theorems on associated transform developed in this paper are rigorous and very useful in performing the inverse Laplace transform for certain functions. These theorems can be applied to directly derive many associated pairs, and thus one can easily extend the tables given in [5]-[7] many fold. Moreover, the results of this paper will help develop more basic theorems in this direction, and will appear in subsequent papers.

ACKNOWLEDGEMENT. The authors express their grateful thanks to the University of Wisconsin at River Falls for providing financial support in publishing this paper.

\section{REFERENCES.}

[1] Volterra, V., Theory of Functionals and of Integral and Integro-differential Equations, Blackie \& Sons, London, 1930.

[2] Wiener, N., Response of a Non-linear Device to Noise, Report 129, Radiation Laboratory, M.I.T., 1942.

[3] Brilliant, M. B., Theory of the Analys is of Nonlinear Systems, Report 345, Research Laboratory of Electronics, M.I.T., 1958.

[4] Barrett, J. F., The Use of Functionals in the Analysis of Nonlinear Physical Systems, J. Electron. Control, Vol. 15, pp. 567-615, 1963.

[5] Lubbock, J. K. and Bansal, V. S., Multidimensional Laplace Transforms for Solution of Nonlinear Equations, Proc. IEE, Vol. 116, No. 12, December 1969, pp. 2075-2082.

[6] Chen, C. F. and Chiu, R. F., New Theorems of Association of Variables in Multiple Dimensional Laplace Transform, Int. J. Systems Sci., Vol. 4, No. 4, 1973, pp. 647-664.

[7] Koh, E. L., Association of Variables in n-dimensional Laplace Transform, Int. J. Systems Sci., Vol. 6, No. 2, 1975, pp. 127-131.

[8] Roberts, G. E. and Kaufman, H., Table of Laplace Transforms, W. B. Sauriutis ", London, 1966. 


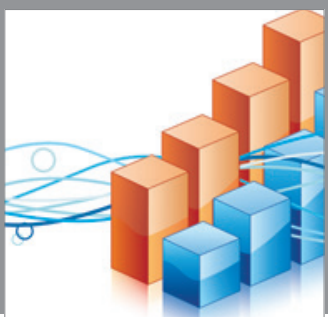

Advances in

Operations Research

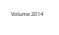

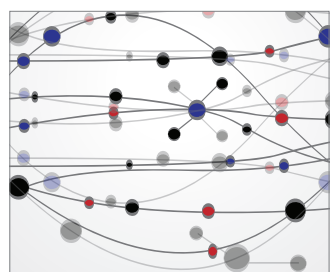

\section{The Scientific} World Journal
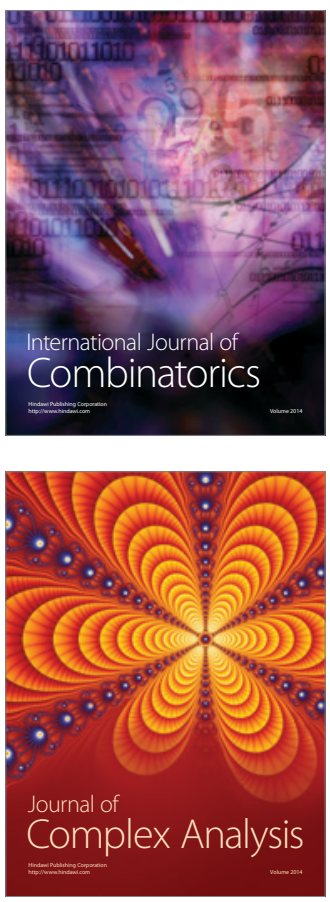

International Journal of

Mathematics and

Mathematical

Sciences
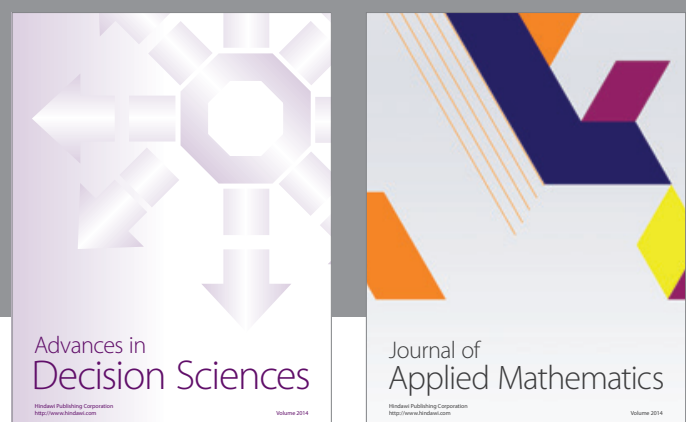

Journal of

Applied Mathematics
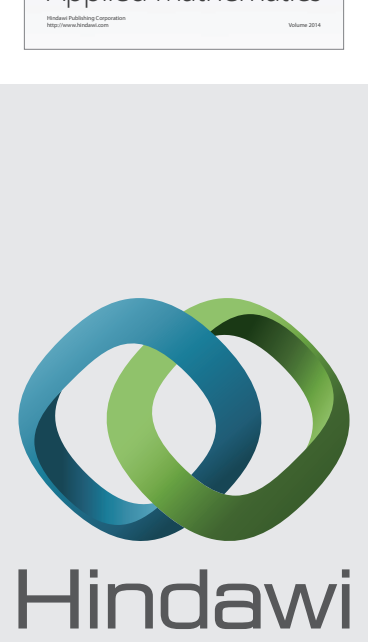

Submit your manuscripts at http://www.hindawi.com
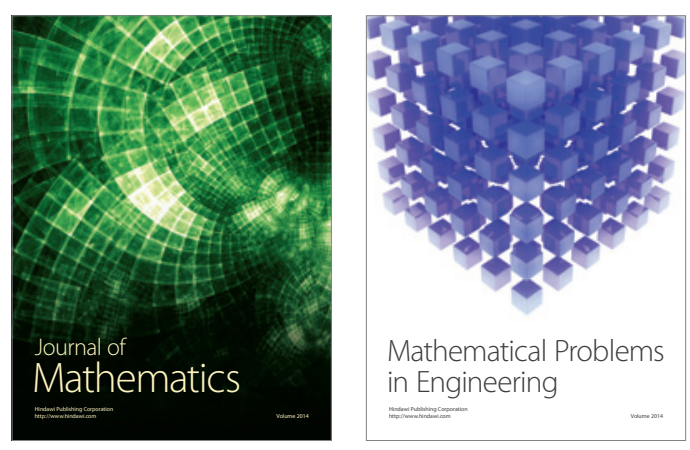

Mathematical Problems in Engineering
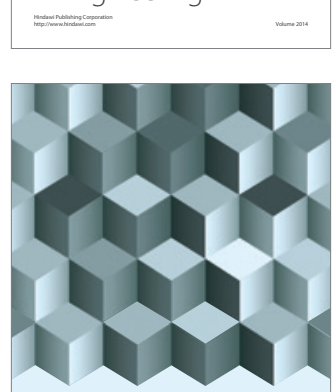

Journal of

Function Spaces
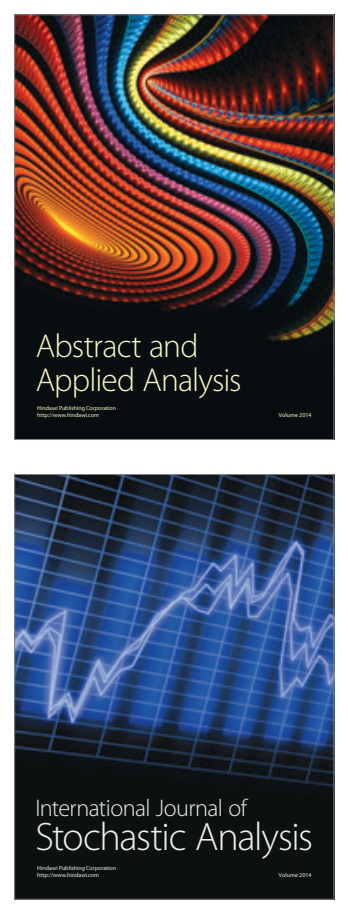

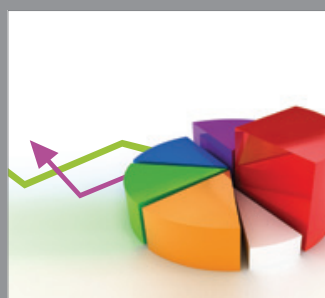

ournal of

Probability and Statistics

Promensencen
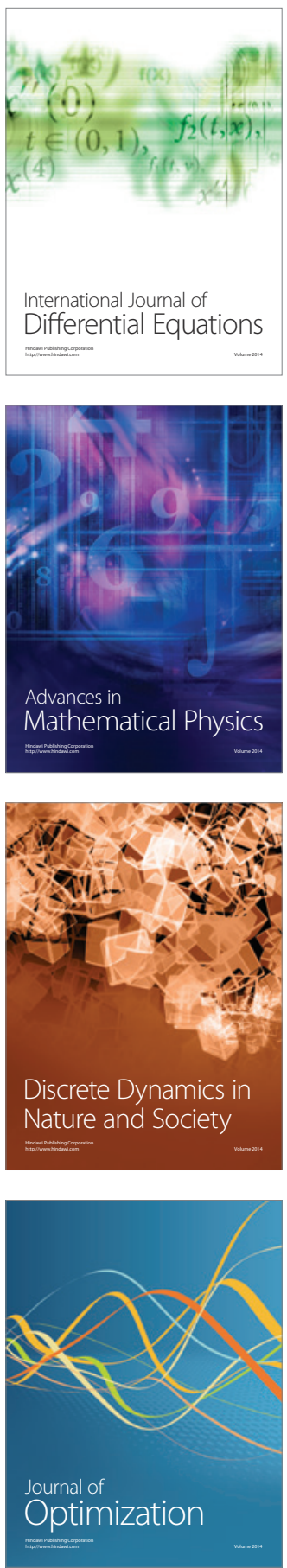